\title{
Kantianismus, Utilitarismus und die Menschenwürde
}

\author{
Werner Wolbert
}

\section{Einleitung}

Anlässlich von möglichen Triage-Problemen im Zuge der CoronaPandemie wie auch in anderen Kontexten werden bisweilen zwei ethische Ansätze gegenübergestellt: eine Ethik der Würde gegen einen utilitaristischen Ansatz. In anderen Kontexten, etwa in Lehrbüchern der Ethik, findet man unter den normativen Theorien häufig „Kantianismus“ als Alternative zum „Utilitarismus“; schließlich verbindet sich die Idee der Menschenwürde vor allem mit dem Namen Kant. Die Idee, dass beides nicht zusammenpasst, ist nach Richard M. Hare die „current orthodoxy“ mindestens seit dem frühen 20. Jahrhundert. ${ }^{1} \mathrm{Da}$ das Wort „Utilitarismus“ im deutschen Sprachraum - spätestens seit Nicolai Hartmann ${ }^{2}$ - mit negativen Konnotationen verbunden ist, scheint sich von der genannten Opposition her eine Ethik der Würde zu empfehlen. Das ist allerdings nicht in jedem Kontext so. So hat man etwa in der Diskussion um die Nutzung embryonaler Stammzellen von der „Ethik der Würde“ eine „Ethik des Heilens" unterschieden und durch diese Terminologie natürlich bereits eine klare Präferenz für die letztere Position zum Ausdruck gebracht. In dieser Distinktion erscheint eine Ethik der Würde eher als eine Art Bremsklotz,

1 Vgl. Hare, Could Kant have been a Utilitarian?, 147-148.

2 Vgl. Hartmann, Ethik, 87: „Den Utilitarismus philosophisch zu kritisieren ist ein leichtes Spiel. Alle seine ungereimten Folgeerscheinungen wurzeln in der banalen Verwechslung von Gut und Nützlich. Das Nützliche ist niemals das Gute im ethischen Sinne.“ Der Utilitarismus mache „die Mittel zum Zweck“ (89). Für Hartmann bezeichnet „nützlich“ immer nur einen Mittelwert, woraus er folgert, Utilitaristen würden nur solche und keine Selbstwerte kennen. Man möchte zweifeln, ob Hartmann je einen Utilitaristen gelesen hat. Und im Übrigen kann das Wort „nützlich“ in der philosophischen Tradition durchaus einen Selbstwert meinen. So sagt Cicero, die Stoiker hätten der These des Sokrates zugestimmt, „ut et, quicquid honestum esse, id utile esse censerent, nec utile quicquam, quod non honestum“ (de officiis III 3, 11). Und (III 30, 110): "Est enim nihil utile, quod idem non honestum, sed quia honestum, utile." Schließlich versucht Platon in der Politeia (vor allem im 1. Buch) zu zeigen, dass Gerechtigkeit nützlich sei. Und eine Schrift Augustins trägt den Titel „De utilitate credendi“. 
der wissenschaftlichen Fortschritt und dessen wohltuende Effekte für die Menschheit behindert. Terminologie ist eben häufig auch ein Stück Sprachpolitik. Dabei geht es dem Utilitarismus (besonders bei Bentham) doch vor allem um Minderung des Leidens (z. B. von Strafgefangenen); insofern könnte auch dieser sich als eine Ethik des Heilens präsentieren. Bisweilen erscheint als Gegensatz zur Ethik der Würde aber auch eine Ethik der Interessen, wobei Erstere den Spielraum der Letzteren einzuengen scheint, während für Letztere Wahlfreiheit und die Vermehrung von Optionen (maximizing choices) ein häufig unhinterfragtes oberstes Ziel zu sein scheint. Ist nun eine Ethik der Interessen eine Spielart des Utilitarismus? Dafür würde sprechen, dass John Stuart Mills Schrift „Über die Freiheit“ (On Liberty) gleichsam die Bibel eines solchen Liberalismus ist. Andererseits hat Verfolgung von Interessen mit Autonomie, Selbstbestimmung zu tun; und diese Stichworte verbinden sich vor allem mit dem Namen Kant.

\section{Zablen}

Ein häufig diskutierter Aufsatz eines amerikanischen Philosophen trägt den Titel „Should the Numbers Count?“3 Dieser Titel könnte als Kernfrage an utilitaristische Ansätze verstanden werden, von denen man häufig annimmt, dass dort nur Zahlen zählen, dass rein quantitativ gedacht wird. Entsprechend gibt es Überlegungen, dass etwa die medizinische Versorgung älterer Menschen viele Ressourcen bindet, die im Kampf gegen die Pandemie vielleicht effektiver eingesetzt werden könnten. ${ }^{4}$ Hier geht es um Zahlen und Quantitäten, Ressourcen und Kosten, Personenzahl, Lebensjahre und besondere Berufe, die für Pflege und Therapie zuständig sind und deren Qualifikation wiederum Auswirkung auf die Zahlen hat. Das Projekt „effektiver Altruismus“ ist ein instruktives Beispiel für solche Positionen. Peter Singer, einer ihrer Proponenten, erwähnt als Beispiel die amerikanische Stiftung Make-a-Wish, die schwerkranken Kindern einen Herzenswunsch erfüllt. Die in einem Fall gespendeten 7.500 \$ wären nach

3 Siehe Taurek, Should the Numbers count?

4 Es gab sogar den Vorschlag, Senioren sollten sich für Versuche mit Impfstoffen zur Verfügung stellen; im Falle einer schweren Schädigung könnten sie ja um Sterbehilfe bitten. Solche Argumente können auch kommunitaristischer Art sein: Im Sinne des Überlebens einer Gemeinschaft haben sich die Älteren für die Jüngeren zu opfern bzw. für solche, die für das Überleben der Gemeinschaft wichtig sind. Derartige Überlegungen gab es z. B. im Judentum als einer Kommunität, deren Überleben gefährdet war. 
Singer besser einer Stiftung, die Malaria bekämpft, zugeteilt worden; die Effizienz, die Zahl der Nutznießer, wäre erheblich höher gewesen. ${ }^{5}$ Schwieriger ist die Sache, wenn mit Hinweis auf die Zahlen durch die entsprechende Maßnahme einer Minderheit zusätzlicher Nachteil oder zusätzliches Leid entsteht, wie im Fall der Idee, durch schwache Schutzmaßnahmen gegen Corona und das In-Kauf-Nehmen von mehr Toten eine Herdenimmunität zu erreichen (falls das möglich wäre). Dagegen wird dann oft in kantischem Vokabular argumentiert, hier würden Menschen zu Mittel gemacht. Prinzipiell ist richtig, dass man so effektiv wie möglich helfen soll, dass möglichst viele Menschen davon profitieren und dass man solche Organisationen unterstützt, die dafür die beste Kompetenz haben etc. Zahlen, so zeigt sich dabei, sind aber nicht immer ein eindeutiges Kriterium. Angesichts der vielen Bittbriefe, die man mit der Post bekommt, stellt sich nicht nur die Frage, welche Organisationen man bedenkt und welche nicht, sondern auch die Alternative - salopp formuliert: Kleckern oder Klotzen? Gibt man an viele wenig, oder bedenkt man wenige reichlich? Effizienter soll letzteres sein, speziell unter der Voraussetzung, dass die Menschen nach unterschiedlichen Präferenzen handeln und damit ihre Wohltätigkeit sich irgendwie verteilt und so vielleicht halbwegs spontan koordiniert wird. Auch wenn sich hinter den Zahlen durchaus ethische Kriterien verbergen, so erscheinen doch in der klassischen utilitaristischen Formel, gemäß der es um „The greatest Happiness of the greatest Number"6 geht, die Interessen oder Rechte der einzelnen Person im Kollektiv aufzugehen. Dabei vergisst man allerdings häufig, dass für Bentham auch gilt: "Everybody to count for one, nobody for more than one."7

5 Vgl. Singer, Most Good, 5f. Singer gibt zu, dass man natürlich lieber für jemanden spendet, der ein Gesicht hat, bei dem man auch die Wirkung seines Tuns sehen kann; so macht eben Wohltun auch Freude. Außerdem stellt sich das Problem nur bei einigermaßen großen Spenden. Singers Thesen sind häufig kontraintuitiv. Dagegen präzise zu argumentieren ist aber auch nicht ganz leicht; diese Gegenargumente müssten nicht in jedem Fall kantisch sein.

6 Bentham, Fragment, 3: das fundamentale Axiom sei: „it is the greatest happiness of the greatest number that is the measure of right and wrong”.

7 So wird Bentham von Mill zitiert; wörtlich findet sich dieses Zitat aber nicht bei Bentham (vgl. „Benthams Dictum“, in: http://www.ethikseite.de/prinzipien/zdictu m.html [28.05.2020]). 


\section{Trümpfe}

Als Korrektur gegen quantitatives Denken beruft man sich bisweilen auf Rechte, welche dieses Denken einschränken sollen. So schlägt etwa Ronald Dworkin vor: „One very practical way to achieve this restriction is provided by the idea of rights as trumps over unrestricted utilitarianism." ${ }^{8}$ In ähnlicher Weise versteht Robert Nozick Rechte im Sinne von Beschränkungen (side constraints) utilitaristischer Überlegungen. ${ }^{9}$ Die Frage ist aber zum einen, welche Art utilitaristischer Überlegungen durch welche Rechte einzuschränken sind. Liberale Denker wie Dworkin und Nozick denken bei Letzteren vor allem an individuelle Präferenzen, subjektive Freiheitsrechte, wie sie schon mit dem Stichwort „Ethik der Interessen“ angedeutet wurden. $\mathrm{Zu}$ deren Rechtfertigung bemüht Nozick auch die kantische Selbstweckformel. Die side constraints sind „Ausdruck des kantischen Grundsatzes, dass die Menschen Zwecke und nicht bloß Mittel sind; sie dürfen nicht ohne ihr Einverständnis für andere Ziele geopfert oder gebraucht werden. Der einzelne ist unverletzlich." ${ }^{10}$ Das ist freilich eine moderne Interpretation der kantischen Formel, die das Einverständnis der Betroffenen zum Kriterium dessen macht, was es heißt, als Selbstzweck behandelt zu werden. Zwar ist auch für Kant Wahlfreiheit von fundamentaler Bedeutung. Die kantische Forderung, der Mensch müsse den Zweck des Handelns anderer „in sich enthalten" 11 , bedeutet aber zunächst, dass dieser als moralisches Wesen, vom Standpunkt der Moral aus zustimmen können muss. Es geht also nicht um eine faktische Zustimmung, sondern um eine normativ verstandene Zustimmung. Nozick scheint dagegen vorauszusetzen, ein Mensch werde zum Mittel gemacht, wenn er faktisch nicht zustimme. Das mag zwar in vielen Fällen stimmen, und faktische Zustimmung ist ethisch höchst relevant; nur leitet sich dieser Gedanke nicht direkt aus der Menschenwürde ab. ${ }^{12}$ Man hat den Eindruck, dass solche Art

8 Dworkin, Rights as Trumps, $162 \mathrm{f}$.

9 Vgl. Nozick, Anarchy, 41.

10 Nozick, Anarchy, 42. Zur kantischen Rede von Zweck und Mittel vgl. Wolbert, Der Mensch als Mittel und Zweck, 71-80.

11 Kant, Grundlegung, IV 430.

12 Vgl. Jones, Kant's Principle of Personality, 56: „To treat a person as a mere means is to treat him as a means to an end in which he cannot, as a rational being, share. It is also to use him as a means to an end which is itself morally unacceptable." Anders im Urteil des deutschen Bundesverfassungsgerichts vom 26.02.2020 zur kommerziellen Sterbehilfe (Siehe: https://www.bundesverfassungsgericht.de/Shar edDocs/Downloads/DE/2020/02/rs20200226_2bvr234715.pdf?_blob=publication File\&v=4). Die Menschenwürde sichere „die Grundbedingungen dafür, dass der 
Kantianismus mindestens auch von Mills Freiheitsschrift inspiriert ist, womit allein schon ein prinzipieller Gegensatz Kant - Utilitarismus fraglich wird. Mill hält allerdings nicht viel von Rechten:

Ich halte es für geraten, dass ich auf jeden Vorteil verzichte, den man für meine Beweisführung aus der Idee eines abstrakten, vom Nützlichkeitsprinzip unabhängigen Rechtes ableiten könnte. Ich betrachte Nützlichkeit [utility] als letzte Berufungsinstanz in allen ethischen Fragen, aber es muss Nützlichkeit im weitesten Sinne sein, begründet in den ewigen Interessen der Menschheit als eines sich entwickelnden Wesens. ${ }^{13}$

Mills zentrales Anliegen betrifft die Möglichkeit der Anwendung von Zwang: „Dass der einzige Zweck, um dessentwillen man Zwang gegen den Willen eines Mitglieds einer zivilisierten Gemeinschaft rechtmäßig ausüben darf, der ist: die Schädigung anderer zu verhüten. "14 Bei Mill ist diese Aussage allerdings auch sozialethisch motiviert. Kant setzt dagegen bei der gleichen Freiheit aller an: Zwang ist nur erlaubt als „als Verhinderung eines Hindernisses der Freiheit“" ${ }^{15}$ Für Mill dagegen ist eine autoritär geprägte und allzu konforme Gesellschaft dem Wohl der Menschen bzw. eines Gemeinwesens abträglich. Entsprechend ist das dritte Kapitel der Freiheitsschrift überschrieben: „Über Individualität als eins der Elemente der Wohlfahrt“.

Positionen wie die von Nozick und Dworkin wären gemäß einer gängigen Unterscheidung in der normativen Ethik als deontologisch einzuordnen, eine Position, für die eine Handlung nicht oder nicht allein durch ihren Beitrag zur Wohlfahrt (well-being) oder zum Nutzen (utility, expediency) zu rechtfertigen ist, wie es eine teleologische (konsequentialistische, pro-

Einzelne seine Identität und Individualität selbstbestimmt finden [...] kann. Namentlich die selbstbestimmte Wahrung der eigenen Persönlichkeit setzt voraus, dass der Mensch über sich nach eigenen Maßstäben verfügen kann und nicht in Lebensformen gedrängt wird, die in unauflösbarem Widerspruch zum eigenen Selbstbild und Selbstverständnis stehen". Hier wären freilich bestimmte Lebensformen, die andere Menschen schädigen (Sadismus, Pädophilie) auszuschließen, womit sich zeigt, dass der Ausdruck der Menschenwürde in diesem Sinne seine Grenzen hat. Die moralische Gesinnung des Menschen, auf die Kant die Würde gründet, kann dagegen nicht in Konkurrenz zu der eines Mitmenschen treten.

13 Mill, Liberty, 37. Der Gedankengang und die zentralen Thesen dieser Schrift sind gut aufgelistet bei Bernd Gräfrath, John Stuart Mill.

14 Mill, Liberty, 35.

15 Kant, Metaphysik der Sitten, VI 231. 
portionalistische) Theorie behaupten würde. ${ }^{16}$ Im Sinne einer Korrektur können dann sowohl bestimmte Rechte oder, in anderen Ansätzen, bestimmte Pflichten dienen, seien diese im präsumtiven Sinn als prima faciePflichten verstanden ${ }^{17}$ oder als absolut geltende Pflichten, wie bisweilen etwa das Verbot der direkten Tötung eines Unschuldigen oder der Falschaussage. ${ }^{18}$ Bei näherer Betrachtung ist aber oft der Unterschied nicht so groß, wenn es konkret wird. Zum einen wird man nicht immer übereinstimmen in der Frage, was denn eine Schädigung ( harm) ist, wie groß denn der Schaden ist oder welche geringeren Schädigungen man bzw. wer gegebenenfalls in Kauf nehmen darf. Zum zweiten kann man die Barriere gegen den „uneingeschränkten Utilitarismus“ unterschiedlich hoch konstruieren, je nach der Größe des zu verhindernden Schadens. Insofern könnte man auch von einem utilitarianism of rights sprechen oder - in anderem Kontext - von einem utilitarianism of duties, der sich spätestens zeigt, wenn ein Konflikt von Rechten oder Pflichten eine Abwägung oder Priorisierung erfordert. Das lässt sich gut illustrieren an der Äußerung von Wolfgang Schäuble über die Priorität des Lebensschutzes, wenn dieser feststellt, dass man zur Rettung von Leben die Wirtschaft nicht langfristig lahmlegen dürfe. Hier deutet sich in der Tat ein schwerwiegender Konflikt an: eine allzu geschwächte Wirtschaft würde auch die Möglichkeiten der Lebensrettung einschränken, weil das Gesundheitssystem nicht mehr finanzierbar wäre. Man hätte also zwischen zwei gravierenden Übeln zu wählen. Hätte Schäuble somit als Utilitarist zu gelten? Das hängt wohl auch vom Zeitpunkt ab, zu dem solche Überlegungen vorgetragen werden. Im letzten Jahr hätte diese Äußerung mit Sicherheit Empörung hervorgerufen und wäre vielleicht als utilitaristisch disqualifiziert worden, in der jetzigen ernsten Situation gab es nur leise Kritik.

16 Was die Terminologie angeht, wird,teleologisch`oft mit,utilitaristisch` gleichgesetzt; häufiger versteht man unter Utilitarismus hingegen eine bestimmte Spielart von Teleologie, vor allem den hedonistischen Utilitarismus von Bentham, Mill und Sidgwick oder den heute gängigen Präferenzutilitarismus etwa von Hare. Paulsen äußert zur Terminologie, er habe seine Position in der ersten Auflage utilitaristisch genannt, wegen dessen historischer Verbindung mit dem Hedonismus habe er aber „mit zu spät kommender Vorsicht, den Ausdruck utilitaristisch durch den Ausdruck teleologisch ersetzt, der zugleich den Vorzug hat, dass er an die allgemeine Weltanschauung erinnert, aus der diese Form der Ethik hervorgegangen ist, nämlich die platonisch-aristotelische" (Paulsen, System der Ethik I, 219).

17 Vgl. Ross, The Right and the Good.

18 Solches Verständnis findet sich außer bei Kant auch in der moraltheologischen Tradition. Vgl. dazu Schüller, Begründung. 


\section{Prinzipien}

Auch Prinzipien werden gelegentlich zu Einschränkung utilitaristischer Überlegungen bemüht. Entsprechend empfehlen Beauchamp und Childress die von ihnen vorgeschlagenen vier Prinzipien: "An advantage of our account is that the principle of utility that we defend can be legitimately constrained by the other principles we advance." 19 Dabei sind die Prinzipien non-maleficience und beneficience auf jeden Fall teleologischer Art; bezüglich des letzteren heißt es denn auch, es sei ,itself an extension of the principle of positive beneficience " 20 , aber, anders als im Utilitarismus nicht das einzige. Die Gerechtigkeit gilt freilich weithin als ein deontologisches Prinzip; aber auch das lässt sich anders deuten wie auch der Respekt vor der Autonomie. Freilich geht es auch einem Utilitaristen wie Mill nicht einfach um kurzfristige Nutzenvermehrung. Auch Mill betont die Nützlichkeit von Prinzipien. Die „überragende Nützlichkeit“ (transcendent expediency) etwa des Verbots der Falschaussage bedeutet für Mill, dass ihre Verletzung weder „um eines kurzfristigen Vorteils für sich selbst oder einen andern willen" ${ }^{21}$ Sinn macht. In diesem Fall sei das Opportune (expedient) nicht mit dem Nützlichen (useful) identisch, sondern ein Teil des Schädlichen. ${ }^{22}$ Allerdings schränkt Mill ein:

Es wird jedoch von allen Ethikern zugestanden, dass selbst diese so unantastbare Regel Ausnahmen zulässt, besonders dann, wenn das Verschweigen einer Wahrheit - indem man etwa einem Übeltäter eine Auskunft verweigert oder einem Schwerkranken eine schlechte Nachricht vorenthält - jemanden, zumal einem andern als sich selbst, vor

19 Beauchamp/Childress, Principles of Biomedical Ethics, 167. Wieso das für die Nicht-Schädigung nicht gelten soll, ist mir nicht einsichtig.

20 Ebd., 166.

21 Mill, Utilitarianism, 69. Birnbacher übersetzt denn auch Expediency unterschiedlich, einmal mit „Opportunismus“, das andere Mal mit „Nützlichkeit“. Vgl. zum Lügenverbot auch Wolf sowie Mills „Utilitarianism“, 101-105. Gray macht in Mill on Liberty noch darauf aufmerksam, dass maximally expedient noch nicht morally required bedeute. Als Letzteres gilt nur, was im Falle des Zuwiderhandelns eine Sanktion nach sich zieht (Strafe, Ächtung durch öffentliche Meinung, Gewissensbisse). Vgl. Mill, Utilitarianism, 145-147: „Hier scheint der eigentliche Angelpunkt für die Unterscheidung zwischen Sittlichkeit und schlichter Nützlichkeit (simple expediency) zu liegen. Es ist allen Formen der Pflicht eigentümlich, dass eine Person zu ihrer Erfüllung rechtmäßig gezwungen werden kann. Pflicht ist etwas, das von jemandem erzwungen werden kann, so wie man die Bezahlung einer Schuld erzwingt."

22 Vgl. Mill, Utilitarianism, 67. 
großem unverschuldeten Unglück bewahrt, und wenn dieses Verschweigen nur durch Ableugnen erfolgen kann. ${ }^{23}$

Mill vertritt somit einen indirekten Utilitarismus mit der Unterscheidung einer kritischen von einer praktischen Ebene, wie ihn ausführlicher Richard M. Hare in seinem Zwei-Ebenen-Ansatz (two level approach) erarbeitet hat. ${ }^{24}$ Solch ein Ansatz rechnet ausdrücklich damit, dass der direkte Bezug auf den Nutzen, das Wohl und Wehe aller Betroffenen, zur Bestimmung der richtigen Handlung diesen Nutzen vereiteln kann, ähnlich wie das ausdrückliche Anstreben von Glück oder Lust dieses Ziel gerade verfehlen kann. Das wird noch deutlicher anhand der schon in obigen Zitaten angedeuteten terminologischen Unterscheidung bei Mill, die im Deutschen schwer wiederzugeben ist. Mill unterscheidet nämlich zwischen $u t i-$ lity und expediency. Das Utility-Prinzip ist zunächst kein normatives, sondern ein axiologisches Prinzip, gemäß dem Glück und Wohlergehen die einzigen intrinsischen Werte sind. ${ }^{25}$ Es ist nicht ein Prinzip, mit dem man direkt die Richtigkeit der Handlung bestimmt; der direkte Bezug auf kurzfristige Folgen und Ziele heißt expediency. Am Schluss seiner Logik formuliert Mill:

I do not mean to assert that the promotion of happiness should itself be the end of all action, or even of all rules of action. It is the justification, and ought to be the controller, of all ends, but is not itself a sole end.” 26 Mit John Gray lässt sich festhalten, „that Mill may consistently allow an important place for moral rules and social norms, which generate obligations and which bar direct appeal to utility, without abandoning his utilitarian commitment. ${ }^{27}$

Das gilt natürlich auch für Rechte als Hindernisse (bars) oder Trümpfe.

23 Mill, Utilitarianism, 69. Im Fall der Schwerkranken denken wir heute etwas anders. Hier würde nur eine Theorie absolut gültiger deontologischer Normen widersprechen, wie man sie bei Kant und in der katholischen moraltheologischen Tradition findet. Letztere hatte in diesem Punkt allerdings genügend Wege einer restriktiven Interpretation des Verbots.

24 Vgl. Hare, Moral Thinking.

25 Die hedonistische Deutung des Glücks durch Mill wäre freilich zu kritisieren; aber das kann hier unberücksichtigt bleiben.

26 Mill, Logic, 621.

27 Gray, Mill on Liberty, 39. 


\section{Konflikte}

Ein Kernproblem deontologischer Theorien ist der Konflikt von Rechten, Trümpfen oder als absolut eingestufte Pflichten. Nach welchen Kriterien soll im Fall der Kollision solcher Rechte oder Pflichten entschieden werden, wenn das teleologische Kriterium des Wohls und Wehes aller Betroffenen auszuschließen ist? Dafür gibt es von deontologischen Ethikern meist keine präzise Antwort; man überlässt sich dabei eher einem nicht näher reflektierten common sense oder einer subjektiven Intuition. Deontologisch verstandene Rechte oder Pflichten gelten für die entsprechenden Autoren meist nur prima facie (auf den ersten Blick). ${ }^{28}$ Von daher versteht sich auch die Beobachtung, dass Utilitaristen und ihre Gegner in konkreten Fragen oft zu denselben Ergebnissen kommen, wenn auch die Gründe dafür unterschiedlich sein mögen. So hält etwa Michael Walzer in Fällen von „supreme emergency“ die gezielte Tötung von Zivilisten im Krieg für möglich: "Given the view of Nazism that I am assuming, the issue takes this form: should I wager this determinate crime (the killing of innocent people) against that immeasurable evil (a Nazi triumph)?”29 Das Flächenbombardement deutscher Städte habe immerhin Ressourcen auf deutscher Seite gebunden, was zu Beginn des Krieges vielleicht die ultima ratio war, später allerdings nicht. ${ }^{30}$ Generell aber gelte: „The deliberate slaughter of innocent men and women cannot be justified simply because it saves the lives of other men and women." 31 . Das muss ein Utilitarist nicht bestreiten, wie wiederum Walzer selbst bemerkt: "I have said that such acts can probably ruled out on utilitarian grounds, but it is also true that utilitarianism as it is commonly understood, indeed, as Sidgwick himself understood it, encourages the bizarre accounting that makes them (morally) possible." 32

Gleichwohl könnte die jeweilige Tradition auch verschiedene Tendenzen oder Präsumtionen favorisieren. Wer sich einem kantischen Ansatz verpflichtet weiß, wird vielleicht zunächst Triage-Überlegungen entschieden von sich weisen. Andererseits sind, falls eine entsprechende Situation

28 Vgl. Mackie, Right-based Moral Theory, 177 und Ross, The Right and the Good.

29 Walzer, Just and Unjust Wars, 259.

30 Vgl. Ebd., 258-263.

31 Ebd. 262. Vgl. ebd.: "There is much else that we might plausibly want to preserve: the quality of our lives, for example, our civilization and morality, our collective abhorrence of murder, even when it seems, as it always does, to serve some purpose."

32 Ebd. Ob diese Einschätzung Sidgwicks zutrifft, sei dahingestellt. 
absehbar ist, solche Überlegungen im Voraus anzustellen und klare Kriterien zu formulieren; andernfalls besteht im Ernstfall die Gefahr schlecht überlegter Ad-hoc-Entscheidungen. Schließlich könnten solche frühzeitigen Überlegungen aber auch das Bemühen um Vermeidung von TriageSituationen schwächen, da man ja schon eine Lösung für solche Konfliktfälle parat hat und so das Gewissen vorzeitig beruhigt sein könnte. Und deswegen mögen teleologische Überlegungen oft voreilig und deswegen suspekt erscheinen, wenn sie gravierende Übel in Kauf nehmen. Für jemanden, der sich von Berufs wegen mit ethischen Fragen befasst, sind freilich derartige zunächst rein hypothetische Überlegungen nicht ungewöhnlich. Für medizinische Praktiker mag das anders aussehen.

\section{Egoismus und Altruismus}

Einen wichtigen Grund für die mit dem Wort „Utilitarismus“ oft gegebene negative Konnotation zeigt Dieter Birnbacher in seinem einschlägigen Artikel auf:

Außerhalb der englischsprachigen Länder wird der Ausdruck >Utilitarismus $<$ und seine Ableitungen vielfach für eine egoistische Verhaltensorientierung verwendet, die den eigenen Nutzen bzw. die Befriedigung eigener Interessen zum ausschließlichen oder überwiegenden Maßstab des Handelns macht. Dieser Sprachgebrauch wird durch die vorherrschende Redeweise von $>$ Nutzen $<$ im Sinne von $>$ Eigennutz $<$ nahe gelegt. ${ }^{33}$

Wer diese Vorstellung von Utilitarismus teilt, wird wohl von der folgenden Äußerung Mills überrascht sein:

Der Utilitarismus fordert von jedem Handelnden, zwischen seinem eigenen Glück und dem der andern mit ebenso strenger Unparteilichkeit zu entscheiden wie ein unbeteiligter und wohlwollender Zuschauer. In der Goldenen Regel, die Jesus von Nazareth aufgestellt hat, finden wir den Geist der Nützlichkeitsethik vollendet ausgesprochen. Die Forderungen, sich dem andern gegenüber so zu verhalten, wie man möchte, dass er sich einem selbst gegenüber verhält, und den Nächs-

33 Birnbacher, Utilitarismus, 96f. 
ten zu lieben wie sich selbst, stellen die utilitaristische Moral in ihrer höchsten Vollkommenheit dar. ${ }^{34}$

Ein Blick in Henry Sidgwicks Methods of Ethics zeigt, dass für diesen der Terminus „Utilitarismus“ im Gegensatz zu Egoismus einerseits und zu Intuitionismus (was hier eine deontologische Theorie meint) andererseits steht, also nichts mit Egoismus zu tun hat. ${ }^{35}$ Hier ist auch auf das persönliche Engagement der Teilnehmer des Projekts „Effektiver Altruismus“ hinzuweisen, die einen festen Teil ihres Einkommens zu spenden pflegen und insofern einen beispielhaften Altruismus praktizieren. ${ }^{36}$ Wer von Utilitarismus - speziell in negativer Konnotation - redet, sollte also jeweils seinen Sprachgebrauch deutlich machen und die egoistische Konnotation ausschließen.

Meistens bezieht man sich mit dem Terminus Utilitarismus auf die hedonistische Variante von Bentham, Mill und Sidgwick (falls man überhaupt einen von diesen gelesen hat). Alle drei Autoren vertreten einen hedonistischen Utilitarismus. Aus der hedonistischen Axiologie ergeben sich gewisse Schwierigkeiten. Wo es um Maximierung des Glücks geht bzw. so vor allem bei Bentham - um Minimierung von Leid, könnte es letztlich schlicht auf eine insgesamt positive Bilanz ankommen. Herbert L. A. Hart (keineswegs ein Antiutilitarist) formuliert als Bedenken, in der Perspektive des klassischen Utilitarismus hätten die verschiedenen Individuen keinen inneren Wert. Man werfe dem Utilitarismus vor, "it treats individuals as mere receptacles with no intrinsic value for the experiences of pleasure and pain". ${ }^{37}$ Bentham erwecke den Eindruck, "as if he was concerned with manipulable and predictable animals or machines - pleasure and pain machines - rather than men". ${ }^{38}$ Das zeige sich etwa in Benthams Kritik der Sklaverei: Diese war "conducted entirely in terms of utility and was based

34 Mill, Utilitarianism, 53. Hare formuliert zu Beginn seines einschlägigen Artikels (Utilitarianism, 640): „Utilitarianism is the extension into philosophy of the Christian doctrine of agape [...], which has counterparts in the various formulation of the Golden Rule that have been preached within all the other higher religions."

35 Zu Sidgwick vgl. Weiß, Deontologie und Teleologie und Wolbert, Vom Nutzen der Gerechtigkeit.

36 Singer spendet nach eigener Aussage ein Drittel.

37 So Hart in der „Introduction“ zu Bentham, Principles of Morals and Legislation, XLVII.

38 Hart, Bentham and Beccaria, 51. Dagegen wisse Beccaria, dem Bentham viel verdankt, sehr wohl um solchen inneren Wert. Bei Beccaria findet sich übrigens schon die Formel vom größten Glück der größten Zahl („la massima felicità divisa nel maggior numero“). 
mainly on the fact that mass slave labour in the end would be found unprofitable to society". ${ }^{39}$ Die Schwierigkeiten mit dem "inneren Wert", der Personenwürde zeigen sich deutlich bei Sidgwick, dessen Position sich (nach Rashdall) auf folgende Weise zusammenfassen lässt: „It is a duty to promote universal good, but universal good is merely pleasure. “40 Damit nimmt Sidgwick gegenüber der Pflicht eine kantianische Haltung ein, während er bezüglich der Idee des Guten eine hedonistische Position vertritt. Bei näherem Nachdenken über das Ziel, das er für andere zu befördern habe, müsse der Mensch zu dem Ergebnis kommen, dass es nur aus der Lust (pleasure) bestehe. Sidgwick teilt somit dem Individuum und der menschlichen Gemeinschaft ein je unterschiedliches Ziel zu. Damit gibt es keine Maxime, die, kantisch gesprochen, zu einem allgemeinen Gesetz werden könnte ${ }^{41}$. Einerseits verlangt die Vernunft von uns, bisweilen in einer Weise zu handeln, die unseren eigenen Interessen entgegensteht (etwa Unrecht zu leiden), nämlich aus der Liebe zum Richtigen und Vernünftigen als solchem; dennoch sollen wir alle anderen Menschen außer uns selbst so behandeln, als seien sie rationalen Begehrens (also der Selbstlosigkeit) unfähig, als begehrten sie vernünftigerweise nichts anderes als die Lust. Bei Sidgwick bleibt die Frage offen, warum man denn vernünftig handeln soll, während für Kant reine Vernunft praktisch ist, d. h. dass der Mensch durch seine Vernunft schon mit der sittlichen Forderung konfrontiert ist. ${ }^{42}$

Wenn nun aber die egoistische und die universalistische Einstellung gleichermaßen als gut und vernünftig gelten, haben die Wörter vernünftig und gut verschiedene Bedeutungen. Für den Egoisten würde vernünftig so viel bedeuten wie: konsistent oder förderlich als Mittel zu dem Ziel, das jeder für sich anstrebt. Wo ich dagegen eine universalistische Einstellung als vernünftig charakterisiere, und wenn diese Vernünftigkeit für mich das Motiv ist, so zu handeln, müsste diese Vernünftigkeit selbst für mich von innerem objektivem Wert sein; das ist die Position eines Ideal Utilitarianism, wie ihn u. a. Rashdall vertritt. ${ }^{43}$ Nur wenn solch vernünftiges Han-

39 Ebd.

40 Rashdall, Theory, I 54: „Reason bids him make duty rather than private pleasure his own end, but in thinking what is the end that he is to promote to other people, it pronounces that end to be pleasure."

41 Rashdall, Theory, I 55.

42 „Reine Vernunft ist für sich allein praktisch und giebt [dem Menschen] ein allgemeines Gesetz, welches wir das Sittengesetz nennen.“ (Kant, Kritik der Praktischen Vernunft, Akademieausgabe V 31).

43 Vgl. Rashdall, Theory, I Kap 7. 
deln in sich gut ist, kann es auch ein größeres Gut sein als das, was ich selbst durch die Suche nach der Lust erhalten könnte. Folgende zwei Dogmen Sidgwicks sind also, wie Rashdall betont ${ }^{44}$, nicht miteinander vereinbar, sie neutralisieren sich gegenseitig:

1. Es ist vernünftig, altruistisch zu sein.

2. Vernünftig zu sein ist nicht etwas in sich Gutes für den, der vernünftig ist, bzw. ist kein innerer Wert.

Die Haltung des guten Menschen in der Interpretation von Sidgwick läßt sich mit Rashdall so erläutern:

I see that it is reasonable for me to prefer my neighbour's good, but this preference has in it nothing intrinsically desirable or beautiful or noble or worth having for its own sake. Duty is duty, but it is not good. Duty is reasonable, but pleasure is better; what the irrational man secures to himself by selfishness is intrinsically better than what the good man gets by obeying the voice of Reason within him. ${ }^{45}$

\section{Menschenwürde und unbedingte Pflicht}

Ein „idealer Utilitarismus“ à la Rashdall würde nun gerade behaupten, dass ein Handeln gemäß der Vernunft, der sittlichen Forderung oder seinem Gewissen selbst innerlich wertvoll ist. Versteht man die sittliche Forderung als unbedingte, kategorische, muss es sich außerdem um den höchsten Wert handeln, zu dessen Realisierung der Mensch unbedingt verpflichtet ist. Diese Unbedingtheit ist möglich, da es allein am Menschen liegt, ob er den Standpunkt der Moral, der Liebe als Wohlwollen und Wohltun, der Goldenen Regel oder den gegenteiligen Standpunkt einnimmt. Diesen intrinsischen und unbedingten Wert nennt Kant „Würde“ im Unterschied zum „Preis“:

Im Reiche der Zwecke hat alles entweder einen Preis, oder eine Würde. Was einen Preis hat, an dessen Stelle kann auch etwas anderes als Äquivalent gesetzt werden; was dagegen über allen Preis erhaben ist, mithin kein Äquivalent verstattet, das hat eine Würde. ${ }^{46}$

44 Rashdall, Theory, I 59.

45 Rashdall, Theory, I 69.

46 Kant, Grundlegung, IV 434. 
Was ein Äquivalent hat, ist austauschbar, ersetzbar. Entsprechend versteht man unter "Utilitarismus" meist eine Ethik, in der alles austauschbar ist, die für alles Äquivalente kennt, für die alle Güter abwägbar sind. Und eine teleologische Ethik ist eben eine Ethik der Güterabwägung, welche aber nicht ausschließen muss, dass es auch einen Wert gibt, der jeder Abwägung entzogen ist, was bei Kant die Würde ist. Kant erläutert: „Also ist Sittlichkeit und die Menschheit, so fern sie derselben fähig ist, dasjenige, was allein Würde hat. “" ${ }^{47}$ Zunächst kommt Würde also der Moralität, der sittlichen Gesinnung zu, dann dem Menschen ${ }^{48}$, sofern er diese realisieren kann, nicht sofern der Mensch die Gesinnung realisiert hat. Im letzteren Fall wären die Menschen nämlich in ihrer Würde ungleich. In der Fähigkeit zur Moralität sind sie dagegen gleich. Und auf Grund dieser Gleichheit gilt Benthams Grundsatz (dessen Begründung aber bei ihm unklar bleibt): Everybody to count for one, nobody for more than one.

Mindestens in der obigen Formulierung Kants erscheint der Wert als ethische Grundkategorie; das ist an sich das Kennzeichen einer teleologischen Ethik. Bekanntlich erscheint allerdings in anderen kantischen Aussagen die Pflicht als solche Grundkategorie; vom griechischen deon (ঠÉov) hat solche Ethik den Namen deontologisch. ${ }^{49}$ Auf der metaethischen Ebene der ethischen Basiskategorie könnte man Kant also sowohl als Deontologen wie als Teleologen einordnen. ${ }^{50}$ An die Bedeutung der Pflicht knüpft das Verständnis von Kant als Erzdeontologen an und als Antipoden gegen jedweden Utilitarismus oder eine Ethik der Güterabwägung. Und Kant selbst hat wohl gedacht, die einzige Alternative zu seinem Ansatz sei irgendeine Form einer hedonistischen Ethik, die keine kategorische Verpflichtung kennt. ${ }^{51}$ Den Gedanken einer unbedingten Pflicht würde aber auch ein idealer Utilitarist bejahen. Das deontische Element „unbedingte Verpflichtung“", das für Moralität konstitutiv ist, ist aber zu unterscheiden

47 Kant, Grundlegung, IV 435.

48 „Menschheit“ bedeutet hier nicht etwa Menschengeschlecht (genus bumanum), sondern das Wesen des Menschen, das, was den Menschen zum Menschen macht (bumanitas).

49 Für heutige konsequentere deontologische Theorien vgl. vor allem Prichard, Does Moral Philosophy rest on a Mistake?; Wolbert, Güterabwägung und Selbstzwecklichkeit.

50 Das Verhältnis beider Bestimmungen wie auch der ersten zur zweiten Fassung des Kategorischen Imperativs ist hier der Kant-Exegese zu überlassen.

51 Vgl. Ewing, Ethics, 60: „He kept thinking of some form of hedonism as the only alternative to his view, and ignored the possibility of a theory which, without taking the hedonist view of good and evil, still derived the obligatoriness or wrongness of an action of its good or bad effects." 
von einer deontologischen Begründung in Einzelfragen des sittlich richtigen Handelns. ${ }^{52}$ Diese Verwechslung findet sich bereits bei Kant selbst, etwa in der Behandlung der Falschaussage oder in seiner Straftheorie. Außerdem wird bisweilen die Idee einer unbedingten Pflicht mit einer ausnahmslos gültigen Pflicht gleichgesetzt. So auch wiederum bei Kant, weil er fälschlicherweise voraussetzt, Ausnahmen erfolgten immer „zum Vortheil unserer Neigung"53; eine Ausnahme kann aber wegen einer vordringlichen Pflicht erforderlich sein. Und diese Ausnahme bedeutet keine Beeinträchtigung der Unbedingtheit und Allgemeingültigkeit der Pflicht, sondern deren Präzisierung in einem konkreten Fall (wie etwa in dem Lehrbuchbeispiel einer Person, die einem Gestapooffizier, der nach versteckten Juden fragt, nicht die Wahrheit sagt). ${ }^{54}$

\section{Conclusio}

Aus den vorgelegten Überlegungen ergeben sich u. a. folgende Konsequenzen:

1. Wer über Utilitarismus spricht bzw. diesen kritisiert, sollte erklären, wie er diesen Terminus versteht bzw. auf welche Version des Utilitarismus er sich bezieht und dabei von den negativen Konnotationen abstrahieren.

52 Vgl. Witschen, Kant als Erzdeontologe, 234.

53 Kant, Grundlegung, IV 424: bei einer Übertretung, so Kant, „nehmen wir uns die Freiheit, für uns oder (auch nur für diesesmal) zum Vortheil unserer Neigung davon [vom allgemeinen Gesetz, Anmerkung] eine Ausnahme zu machen“. Vgl. dazu Rashdall, I 115: „He does not see that the rule ,Do this except in such and such circumstances' is just as ,categorical' and just as little ,hypothetical' as the rule ,Do this under all circumstances whatsoever,' so long as the exceptions are recognized as no less universal in their application, no less based upon the reason and nature of things, than the original rule. Kant in fact confuses the inclusion of an exception in a moral rule with the admission of an exception to a moral rule."

54 Dagegen Kant, Über eine vermeintes Recht (VIII 426): „Wahrhaftigkeit in Aussagen, die man nicht umgehen kann, ist formale Pflicht des Menschen gegen Jeden, es mag ihm oder einem Anderen daraus noch so ein großer Nachtheil erwachsen“. Das ist ein klar deontologisches Verbot. Solche Deontologie ist wohl eher ein traditionelles Relikt; sie muss nicht als für die kantische Ethik konstitutiv gelten. Vgl. hier bes. Cummiskey, Kantian Consequentialism sowie Thilly, Kant and Teleological Ethics. 
2. Nur nach solcher Klärung lässt sich klären, ob die Einwände und Gegenargumente tatsächlich nicht-utilitaristischer oder nicht-teleologischer Art sind.

3. Kant ist nicht der Erzdeontologe, als der er meistens präsentiert wird. Eine von Kant inspirierte Ethik ist kompatibel mit einer teleologischen Theorie, für die Liebe als Wohlwollen und Wohltun das einzige Kriterium sittlich richtigen Handelns ist. Rashdall formuliert: „At its highest the sense of duty is identical with the rational love of persons (including in due measure self-love), and the things which constitute their true good." 55 Zwar gibt es bei Kant deontologische Elemente, vor allem die rigoristischen Positionen zu Lüge, Suizid und Todesstrafe. Andererseits hat er mit einem wirklichen Erzdeontologen wie Prichard wenig gemein. So kann man mit Hare urteilen: „Kant [...] could have been a utilitarian, though he was not." 56

\section{Literatur}

Beauchamp, Tom L./Childress, James F.: Principles of Biomedical Ethics, Oxford: Oxford University Press ${ }^{5} 2001$.

Bentham, Jeremy: An Introduction into the Principles of Morals and Legislation, ed. by J.H. Burns/H.L.A. Hart, London/New York: Methuen 1982.

Bentham, Jeremy: Fragment on Government, ed. by J.H. Burns /H.L.A. Hart, Cambridge: Cambridge University Press 1988.

Birnbacher, Dieter: Art. Utilitarismus, in: Düwell, Marcus/Hübenthal, Christoph/ Werner, Micha H. (Hg.): Handbuch Ethik, Stuttgart: J.B. Metzler ${ }^{3} 2011$, 95-107.

Cummiskey, David: Kantian Consequentialism, New York/Oxford: Oxford University Press 1996.

Ewing, Alfred Cyril: Ethics, New York: Free Press 1953.

Frankena, William K.: Ethics, Englewood Cliffs (New Jersey): Prentice Hall ${ }^{2} 1973$.

Gräfrath, Bernd: John Stuart Mill, „Über die Freiheit“. Ein einführender Kommentar, Paderborn/Wien: Schöningh 1992.

Gray, John: Mill on Liberty. A Defence, London/New York: Routledge ${ }^{2} 1996$.

Hare, Richard Mervin: Moral Thinking. Its Levels, Methods and Point, Oxford: Clarendon Press 1981.

Hare, Richard Mervin: "Utilitarianism", in: Macquarrie, John/Childress James (Eds.): A New Dictionary of Christian Ethics, London: SCM Press 1986, 640643.

55 Rashdall, Theory, I 128.

56 Hare, Could Kant have been a Utilitarian?, 148. 
Hare, Richard Mervin: Could Kant have been a Utilitarian?, in: Ders.: Sorting out Ethics, Oxford: Clarendon Press 1997, 147-165.

Hart, Herbert L.A.: Bentham and Beccaria, in: Ders.: Essays on Bentham. Jurisprudence and Political Theory, Oxford: Clarendon Press 1982, 40-52.

Hartmann, Nicolai: Ethik, Berlin: de Gruyter ${ }^{4} 1962$.

Jones, Hardy E.: Kant's Principle of Personality, Madison: University of Wisconsin Press 1971.

Kant, Immanuel: Gesammelte Schriften, hrsg. von der Königlich Preußischen Akademie der Wissenschaften, Berlin 1902ff. [Akademie Ausgabe: AA].

Kant, Immanuel: Grundlegung zur Metaphysik der Sitten, in: AA Bd. IV, 385-463. [= GMS $]$.

Kant, Immanuel: Kritik der praktischen Vernunft, in: AA Bd. V, 1-163. [= KpV].

Kant, Immanuel: Über ein vermeintes Recht aus Menschenliebe zu lügen, in: AA Bd. VIII.

Mackie, John Lesley: Can there be a Right-based Moral Theory, in: Waldron, Jeremy (Hg.): Theories of Rights, Oxford: Oxford University Press 1984, 168-181.

Mill, John Stuart: Utilitarianism/Der Utilitarismus, übersetzt und herausgegeben von Dieter Birnbacher, Stuttgart: Reclam ${ }^{2} 2006$.

Mill, John Stuart: On Liberty/Über die Freiheit, übersetzt von Bruno Lemke, Stuttgart: Reclam 2009.

Mill, John Stuart: A System of Logic, London: Longmans, Green and Co ${ }^{8} 1959$.

Nozick, Robert: Anarchy, State and Utopia, Malden, MA usw.: Blackwell 1974. (Deutsch: Anarchie, Staat, Utopia, München: Olzog 2011).

Paulsen, Friedrich: System der Ethik mit einem Umriß der Staats- und Gesellschaftslehre, Stuttgart/Berlin: Cotta, ${ }^{6} 1903$.

Prichard, Harold Arthur: Does Moral Philosophy Rest on a Mistake?, in: Ders.: Moral Obligation. Essays and Lectures, Oxford: Clarendon ${ }^{4} 1971$.

Rashdall, Hastings: The Theory of Good and Evil, New York: Kraus Reprint 1971.

Ross, William David: The Right and the Good, Oxford: Clarendon Press 1946.

Schüller, Bruno: Die Begründung sittlicher Urteile. Typen ethischer Argumentation in der Moraltheologie, Düsseldorf: Patmos ${ }^{3} 1987$.

Sidgwick, Henry: The Methods of Ethics, Indianapolis/Cambridge: Hacket ${ }^{7} 1981$.

Singer, Peter: The Most Good You Can Do: How effective altruism is changing ideas about living ethically, New Haven/London: Yale University Press 2015.

Taurek, John M.: Should the numbers count?, in: Philosophy and Public Affairs 6 (1977) 293-316.

Thilly, Frank: Kant and Teleological Ethics, in: Kantstudien 8 (1904) 13-46.

Walzer, Michael: Just and Unjust Wars. A moral Argument with Historical Illustrations, Harmondsworth: Penguin Books 1980. 
Weiß, Andreas-Michael: Deontologie und Teleologie. Einige Definitionen und Klärungen, in: Holderegger Adrian/Wolbert Werner (Hg.): Deontologie - Teleologie, Freiburg i. Ue.: Academic Press/Freiburg im Breisgau: Herder 2012 (= Studien zur theologischen Ethik 135), 15-44.

Witschen, Dieter: Gerechtigkeit und teleologische Ethik, Freiburg i.Ue.: Universitätsverlag/Freiburg im Breisgau: Herder 1992 (= Studien zur theologischen Ethik 39).

Witschen, Dieter: Warum gilt Kant als Erzdeontologe? in: Holderegger Adrian/ Wolbert Werner (Hg.): Deontologie - Teleologie, Freiburg i. Ue.: Academic Press/Freiburg im Breisgau: Herder 2012 (= Studien zur theologischen Ethik 135), 227-247.

Witschen, Dieter: Was verdient moralisch den Vorzug? Ethisches Abwägen durch Präferenzregeln, Stuttgart: Kohlhammer 2017.

Wolbert, Werner: Der Mensch als Mittel und Zweck. Die Idee der Menschenwürde in normativer Ethik und Metaethik, Münster: Aschendorf 1987 (= Münsterische Beiträge zur Theologie Band 53).

Wolbert, Werner: Vom Nutzen der Gerechtigkeit. Zur Diskussion um Utilitarismus und teleologische Theorie, Freiburg i.Ue.: Universitätsverlag/Freiburg im Breisgau: Herder 1992 (= Studien zur theologischen Ethik 44).

Wolbert, Werner: Güterabwägung und Selbstzwecklichkeit. Zur Eigenart einer deontologischen Theorie und zur tugendethischen Alternative, in: Freiburger Zeitschrift für Philosophie und Theologie 52 (2005) 145-170.

Wolbert, Werner: Menschenwürde und Autonomie. Theologisch-ethische Dimensionen, in: Baranzke Heike/Duttge Gunnar (Hg.): Autonomie und Würde. Leitprinzipien in Bioethik und Medizinrecht, Würzburg: Königshausen \& Neumann 2013, 77-95.

Wolf, Jean-Claude: John Stuart Mills „Utilitarismus“. Ein kritischer Kommentar, Freiburg: Alber 1992. 